\title{
Study of Attenuation in Vegetation Media and Prediction Model at Microwave Frequencies
}

\author{
Mahmoud Elshayeb", Silvello Betti \\ Department of Electronic Engineering, University of Rome Tor Vergata, Rome, Italy \\ Email address: \\ elshayebmahmoud@yahoo.com (M. Elshayeb) \\ ${ }^{*}$ Corresponding author
}

To cite this article:

Mahmoud Elshayeb, Silvello Betti. Study of Attenuation in Vegetation Media and Prediction Model at Microwave Frequencies. Science Journal of Circuits, Systems and Signal Processing. Vol. 7, No. 1, 2018, pp. 1-7. doi: 10.11648/j.cssp.20180701.11

Received: November 7, 2017; Accepted: December 5, 2017; Published: January 5, 2018

\begin{abstract}
This paper presents the First report of an ongoing to study the effects of trees on radio signals at microwave frequencies. The effects of vegetation media on the planning and design of radio links at microwave and Microwave frequencies are considerable and must be accounted for by radio system operators and users. Measurements have been made to determine the extent of attenuation when propagated through vegetation and also have been carried to be conducted on selected vegetation with various degree of foliation to determine the dependence of losses on vegetation. Also, path geometry and tree geometry are among other parameters under consideration. The results from these experiments compared with standard ITUR$\mathrm{P}$ empirical models and to reflect the dependence of vegetation attenuation on the input parameters under observation.
\end{abstract}

Keywords: Vegetation, Attenuation, Foliage Depth

\section{Introduction}

Vegetation and trees planted at strategic places all over Italy and at the same time maintain a greener environment. However, their presence may have an adverse effect on telecommunication services as they may cause blockages to radio path by obstructing the line of sight between transmitter and receiver.

As a result, propagating radio waves are forced to follow different paths to the receiver and this situation leads to signal degradation. Removing all trees obstructing line of sight is an impractical solution, but fade mitigation techniques such as adaptive coding and path modulation, path diversity etc. can be adapted to mitigate the effect. So, for radio planners whose aim is to achieve effective communication with high reliability, link availability and good quality of service (QoS), the effect of vegetation has to be taken into consideration during the planning and design work Trees, either singly or as a group influence the level of the signal directly by providing an additional attenuation over free space loss. They act as obstacles causing absorption and scattering to radio signals. When a single tree appears along radio path, it introduces a partial blockage to a line of sight resulting in loss of signal at the receiver end. Considerable efforts have been made in the recent past by various authors Al-Nuaimi et al. (1994, 1998) [1] [2], Ndzi et al. (2005) [3], and Meng et al (2009) [4] to properly estimate the influence of vegetation on radio waves. This has actually stimulated and attracted series of experimental campaigns which led to models for the prediction of attenuation. Apart from signal attenuation, fading is another phenomenon commonly encountered by radio waves propagating through vegetation. Fading in a wireless channel is simply a variation in amplitude and phase level of the received signal. The variation can be due to multipath propagation. When radio signals encounter clutter (e.g vegetation) it may lead to diffraction, reflection, and scattering along the propagation path. Such signals are then forced to follow different (multiple) paths to the destination. So, various subcomponents of the waves would arrive at the receiver at different times. Vegetation plays a significant role in fading a phenomenon in wireless communication. Concerted efforts have been made in the past by Song Meng et al. (2009) [5] and Cheffena et al. (2009) [6] to model path loss at various 
frequencies both analytically and empirically. In a more general term, radio waves obstructed by vegetation are decomposed into various sub-component parts and each forming a propagation mechanism. These are [11]:

Reflection from ground
Diffraction around edges

Diffraction over the tree top

Diffusion and scattering through vegetation. Reflection from other nearby obstacles.

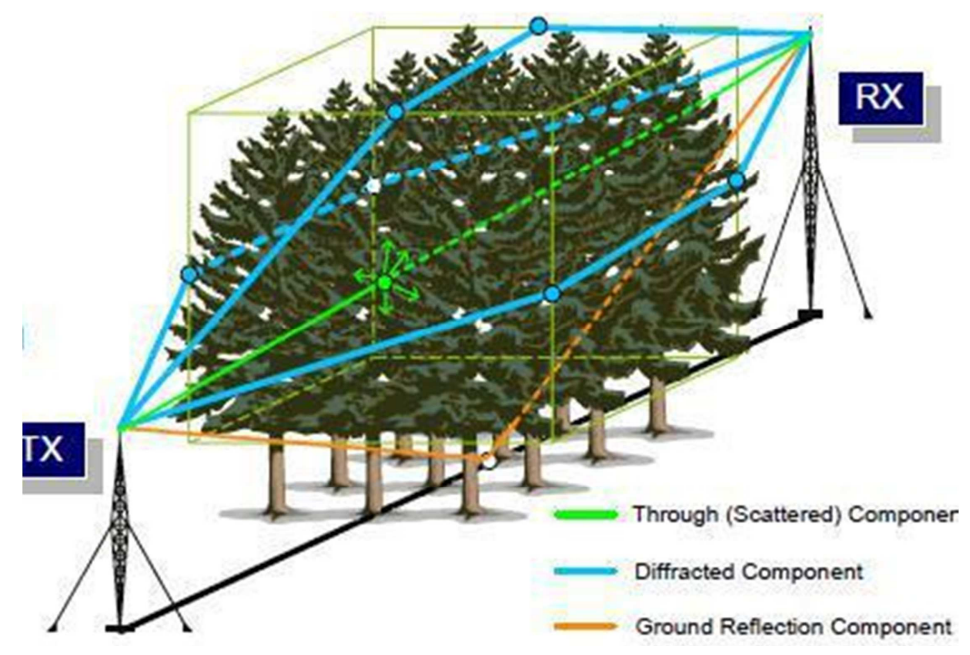

Figure 1. Generic propagation mechanism.

\section{Empirical Attenuation Models}

An empirical model known as the Exponential Decay Model (EXD) was generally used to predict excessive propagation loss in vegetation. From the 1960s, work has been done to discuss the effect of vegetation on Radio waves. Tamir (1967) [7] proposed a half-space model to deal with radio wave propagated in the forest over a large depth of more than $1000 \mathrm{~m}$. Weissberger (1982) [8] proposed the modified exponential decay model (MED) for propagation path blocked by dense, dry, leafed trees found in a temperate climate. The model covers frequencies from $230 \mathrm{MHz}-95$ $\mathrm{GHz}$. The predicted loss is as

$$
\begin{gathered}
L(d B)=1.33 f^{0.284} d_{f}^{0.588} \quad 14 \leq d_{f} \leq 400 m \\
L(d B)=0.45 f^{0.284} d_{f}^{0.588} \quad 0 \leq d_{f} \leq 14 m
\end{gathered}
$$

Where $\mathrm{f}$ is in $\mathrm{GHz}$ and $d_{f}$ is in meters. This parametric equation is seen to have a general format

$$
L(d B)=x f^{y} d_{f}^{z}
$$

Where $\mathrm{X}, \mathrm{y}$, and $\mathrm{z}$ are variables of fitted values obtained from measurements. Following this trend, the international telecommunication union (ITU) in 1986 [9] developed a model for foliage attenuation called early ITU model is given by

$$
L(d B)=0.2 f^{0.3} d_{f}^{0.6}
$$

Where $f$ is in $\mathrm{MHz}$ and $d_{f}$ is in meters. Al-Nuaimi et-al (1994) presented a modified version of equation 3 called modified ITU-R model. This caters for both in-leaf and outof-leaf cases at 11.2GHz and are presented in (4) and (5)

$$
\begin{aligned}
& l(d B)=11.93 d_{f}^{0.398} \quad \text { in }- \text { leaf } \\
& l(d B)=\left\{\begin{array}{c}
1.75 d_{f}, \quad d \leq 31 \mathrm{~m} \\
28.1 d_{f} d>31 \mathrm{~m}
\end{array}\right. \text { out of leaf }
\end{aligned}
$$

A more generalized model above is the fitted ITU-R model (FITU-R) proposed by same authors (Al-Nuaimi et al 1998). It is applicable in the frequency range of $10-40 \mathrm{GHz}$ and presented as

$$
\begin{aligned}
& L(d B)=0.39 f^{0.39} d_{f}^{0.25} \quad \text { in-leaf } \\
& L(d B)=0.37 f^{0.18} d_{f}^{0.59} \text { out-of-leaf }
\end{aligned}
$$

In furtherance of this, COST 235 model [10] was proposed based on measurements conducted on a grove of trees at mill metric frequencies $(9.5 \mathrm{GHz}-57.6 \mathrm{GHz})$ over a depth of less than $200 \mathrm{~m}$ for in-leaf and out-of-leaf. In the empirical models, it is clear that radio waves obstructed by vegetation suffer some losses in excess of free space which are frequency and foliage depth dependence. Having reviewed these models and observed their drawbacks, possible areas of improvements have been identified. For example, none of the models has predicted for partial foliation stage. Whereas, three foliation stages have been identified in vegetation (full leaf, out-of-leaf and partial foliation). All existing models predicted for in-leaf and out-of-leaf. This leaves a gap to be filled. Also, empirical models do not include path geometry in their prediction. Measurement geometry and tree geometry are very important in estimating propagation loss in a vegetative channel.

\section{Proposed Methodology}

Many numbers of experimental have been designed to be 
conducted. The important components factored at the experiments are path geometry, different foliation stages, different tree types and antenna heights. Propagation carried out at different scenarios in the presence of vegetation. A repeat of same experiments conducted without vegetation blockage to model free space.

\subsection{First Measurement}

Equipment Description

The transmitter section is consists of Anritsu MG3692B signal generator with a maximum operating frequency of 20GHz. A discone broadband antenna and an adjustable antenna mast of up to $6 \mathrm{~m}$ to realize varying antenna height. The transmitter is powered by a $230 \mathrm{~V}$ AC taken from a combination of two (2) 12V DC battery source (connected in parallel) and a pure sine wave inverter at $1000 \mathrm{~W}$. The signal generator can generate a continuous wave $(\mathrm{CW}) \mathrm{RF}$ signal up to a maximum power of $+30 \mathrm{dBm}(1 \mathrm{w})$ and be fed through a discone antenna to the receiver. The receiver is made up of Agilent E4440A PSA series spectrum analyzer and a broadband discone antenna (omnidirectional). This is equally powered by a $230 \mathrm{~V} A C$ and taken from two (2) $12 \mathrm{~V}$ DC batteries connected in parallel with a full sine wave inverter. The analyzer has a working frequency range of $3 \mathrm{MHz}$ to $26.5 \mathrm{GHz}$

\subsection{Test Site}

Measurements carried at three different locations for inleaf, out-of-leaf and partial defoliation states.

Transmitting and receiving types of equipment were each arranged on a separate trolley for easy carry to the measurement site.
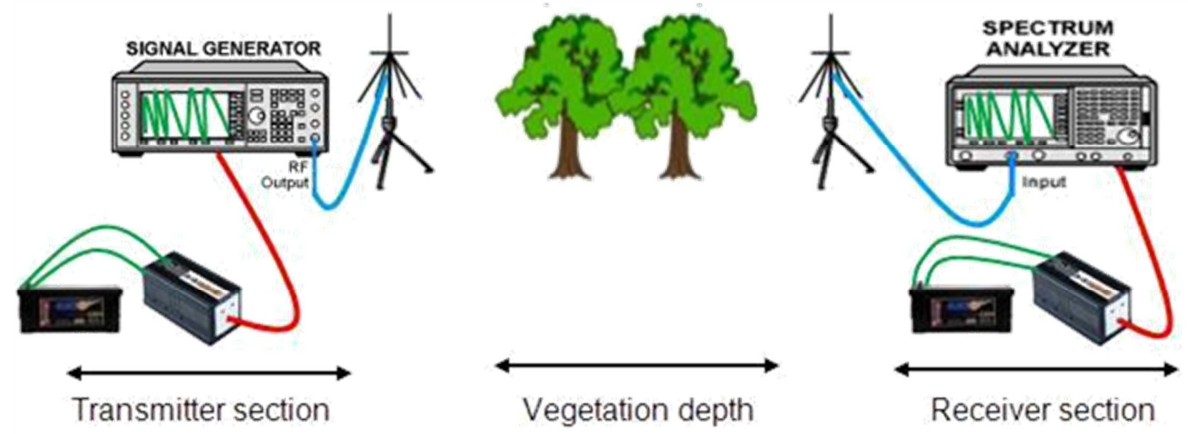

Figure 2. Experimental setup.

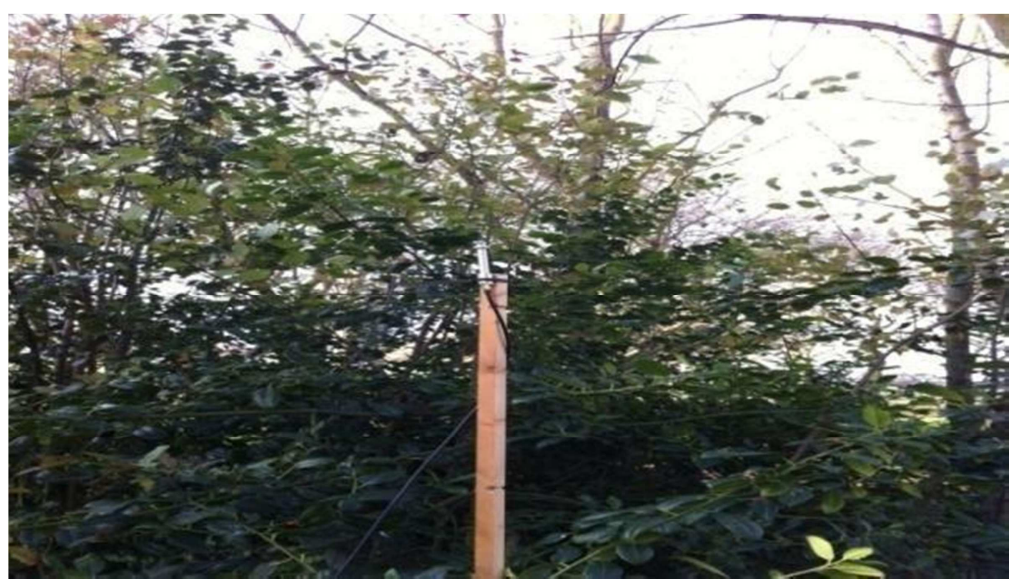

Figure 3. Site one (Full leaf vegetation.

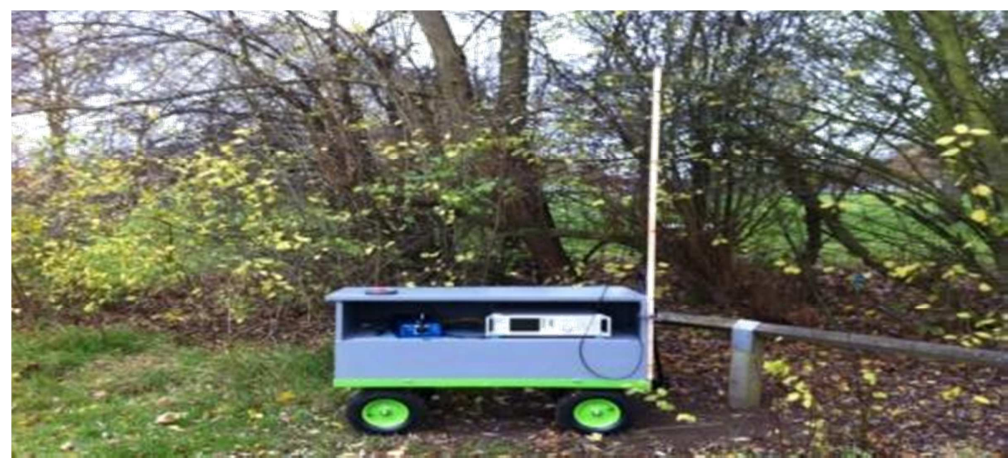

Figure 4. Site two (Partial foliation vegetation). 


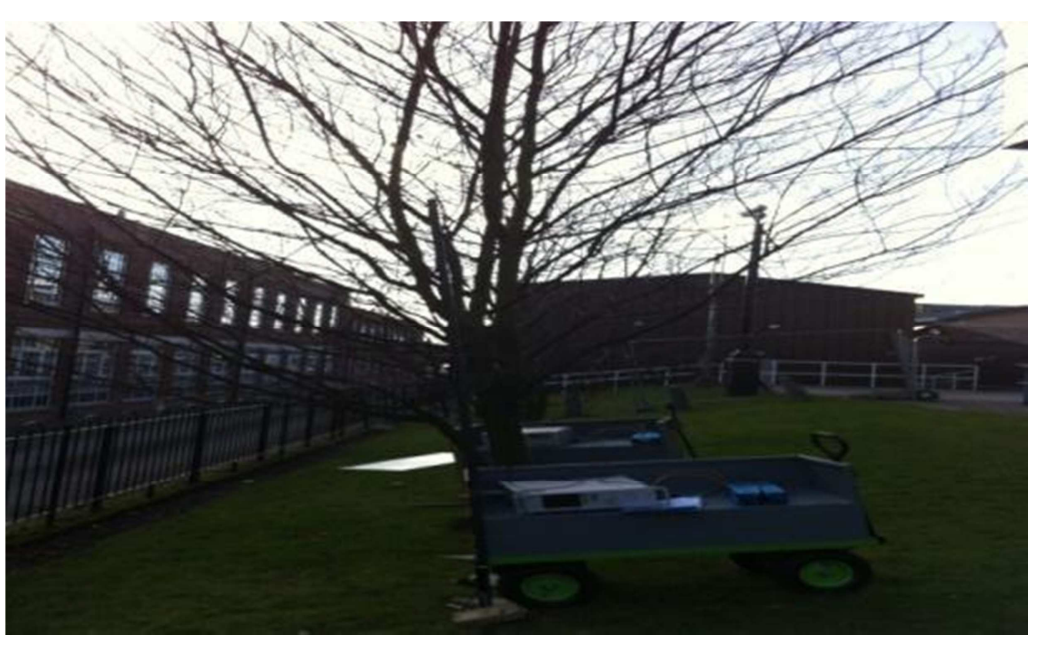

Figure 5. Site three (out-of-leaf).

\section{Result}

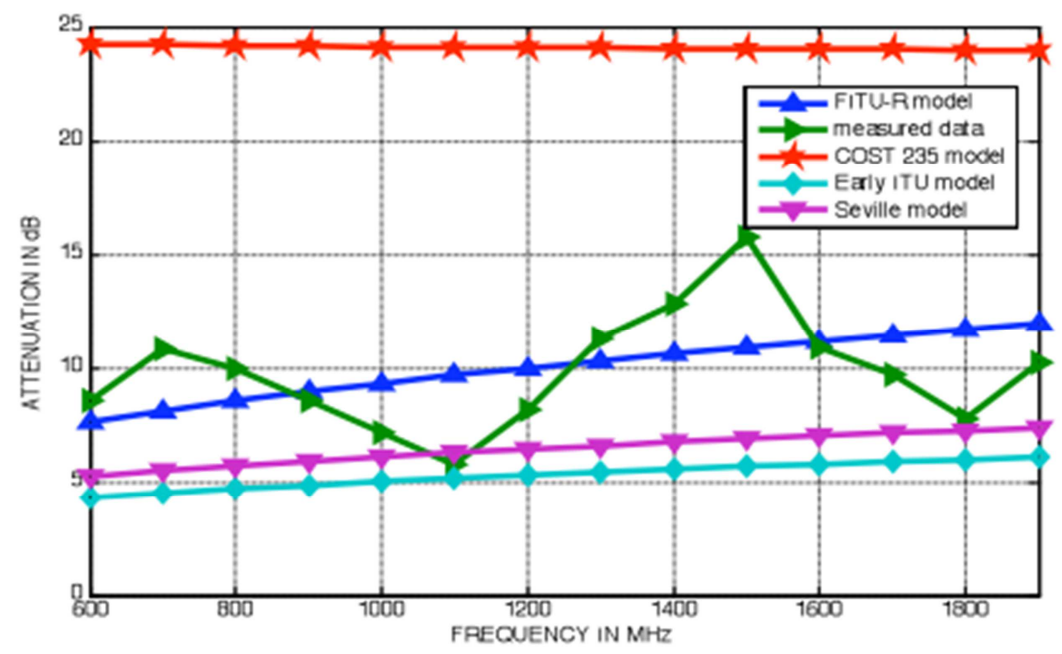

Figure 6. Measured data at foliage depth of $6.8 \mathrm{~m}$ compared with empirical models. (Full leaf).

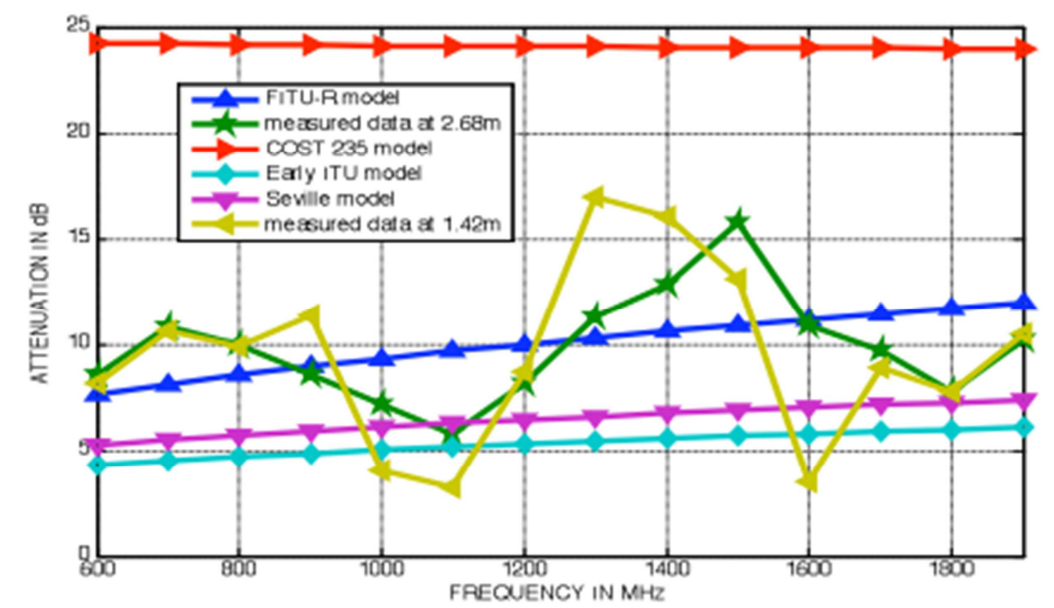

Figure 7. Measured data at two different antenna heights for foliage depth of 6.8m. (full leaf). 


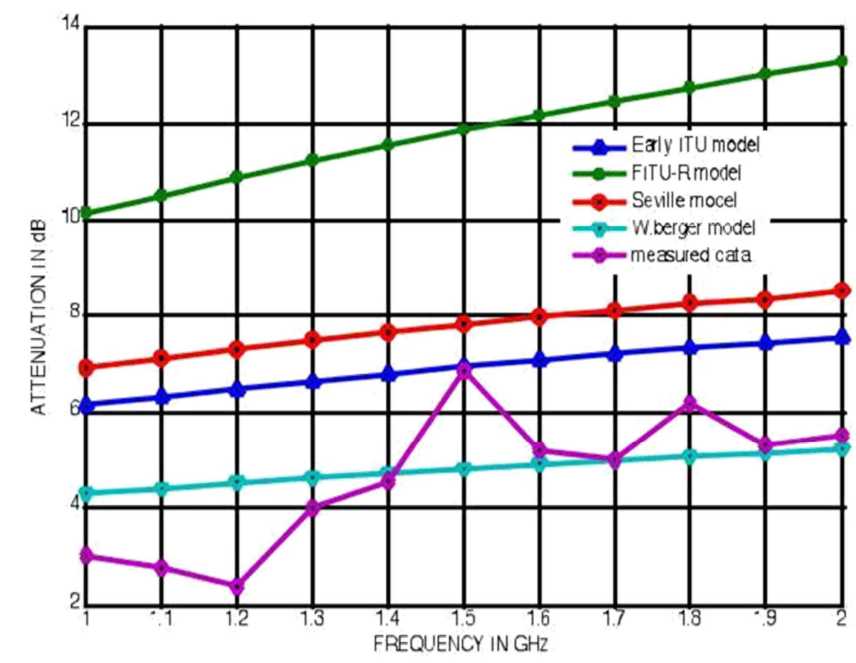

Figure 8. Measured data for partial defoliation compared with empirical models. Foliage depth $=9.5 \mathrm{~m}$.

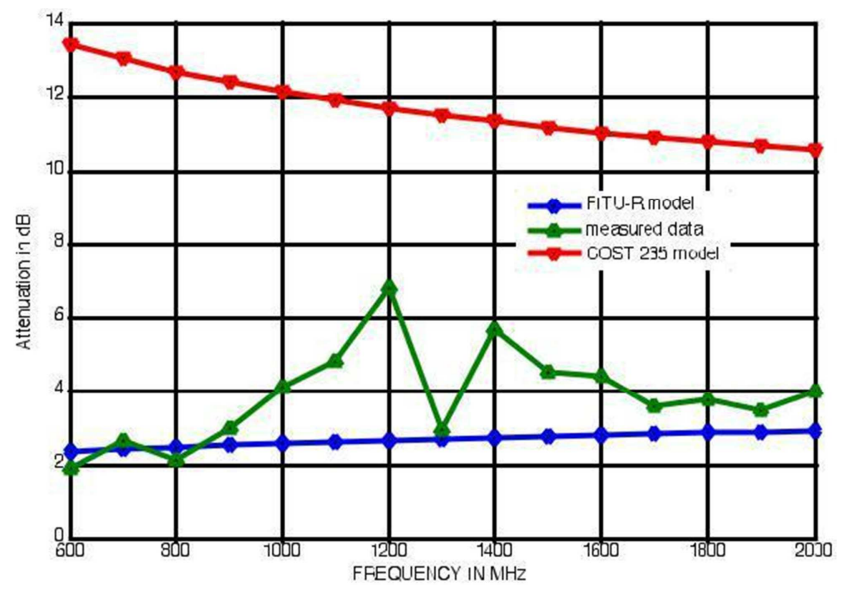

Figure 9. Out of leaf data at a depth of $3.3 \mathrm{~m}$.

\section{Modelling Prediction for Losses}

The modelling of the forest of loss of propagation is very complex and complex as demonstrated by the experimental data. The complexity of modelling is derived from variations in operational contexts and physical parameters of vegetation, such as tree type, tree density, leaf size, leaf density, and measurement geometry, the height of the antenna, etc. Moreover, the characterization of each of these parameters is very deterministic.

However, the need has been felt to have an integral loss prediction model for accurate and efficient planning of wireless communications in vegetation. The transmission loss in a typical forest or forest can be broken down into different components such as

$$
L_{T}(d B)=L_{f s}+L_{v e g}+L_{s y}
$$

Where $L_{T}$ Total channel loss, $L_{F S}$ free space loss, $L_{v e g}$ vegetation loss and $L_{s y}$ system loss.

$L_{v e g}$ in this case is the loss due to only vegetation which contains passive elements that causes physical obstruction to LOS propagation. After the extraction process, Equation (8) is now reduced to

$$
L_{v e g}=\left[L(x)_{T-} L(x)_{f s}\right]
$$

$L(x)_{T}, L(x)_{f s}$ are vectors representing the measured loss in woodland and in an open grassy field respectively at observation points $x$ (where $x=1,2,3,4,5, \ldots \mathrm{n}$ ). The signal power decays with the depth of vegetation. A power law function has been used in the modelling for channel characterization as in

$$
L(d B)=a K^{b}
$$

Where $\mathrm{k}$ represents the parameter being studied, for example, the depth of the vegetation $d_{f} \mathrm{a}$ and $\mathrm{b}$ are least squares variables that must be optimized well to give the best fit to the experimental data. To arrive at the model that will be of more general use, the combined data of different sites and routes with similar operating contexts were combined and average values were taken. All these species are found in two types of propagation into woodland and propagation inside woodland, as shown in Figures 10 and 11. Getting the best fit out of this combined data would reduce site dependent anomalies and make the resulting prediction model. 
The resulting parametric equations that best describe the fitted curves are:

$$
\begin{aligned}
& L(d B)=5,53 d_{f}^{0,37} \text { (At 3,5 GHz) Inside Geometry } \\
& L(d B)=14.49 d_{f}^{0.13} \text { (At } 3,5 \mathrm{GHz} \text { ) Into Geometry }
\end{aligned}
$$

From the parametric equations of 11 and 12 a new prediction model has been developed using the generic empirical prediction format given as

$$
L(d B)=x f^{y} d_{f}^{z}
$$

Values for $\mathrm{x}, \mathrm{y}$ and $\mathrm{z}$ in Equation 13 have been obtained for both into and inside geometries (Table 1) and the resulting equations are as in 14 and 15.

$$
\begin{gathered}
L(d B)=0.56 f^{0.39} d_{f}^{0.15} \text { into woodland } \\
L(d B)=0.28 f^{0.39} d_{f}^{0.31} \text { inside woodland }
\end{gathered}
$$

Table 1. Parameter values for $x, y$ and $z$.

\begin{tabular}{llll}
\hline Geometry & $\mathbf{x}$ & $\mathbf{y}$ & $\mathbf{z}$ \\
\hline Inside & 0.28 & 0.39 & 0.31 \\
Into & 0.56 & 0,39 & 0,15 \\
\hline
\end{tabular}

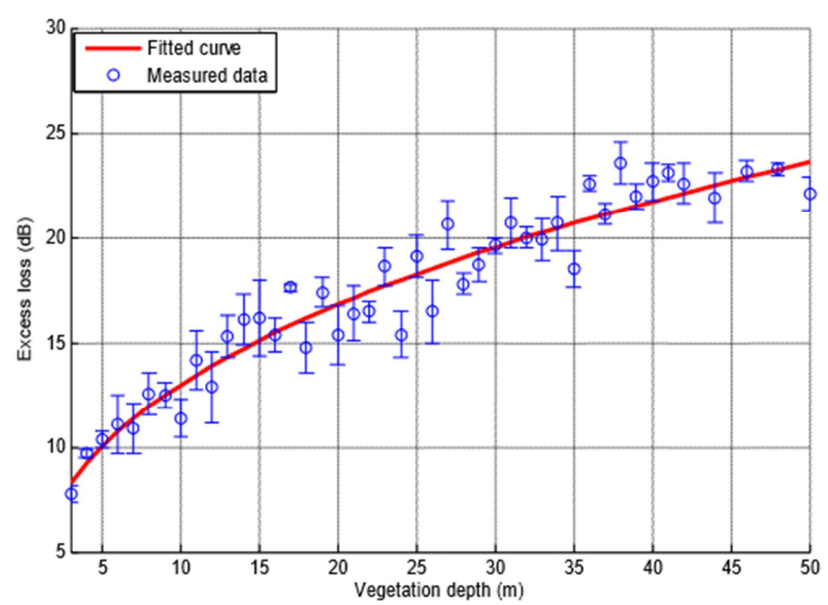

Figure 10. Excess loss for 'inside geometry' fitted with a curve using the combined data at $3.5 \mathrm{GHz}$.

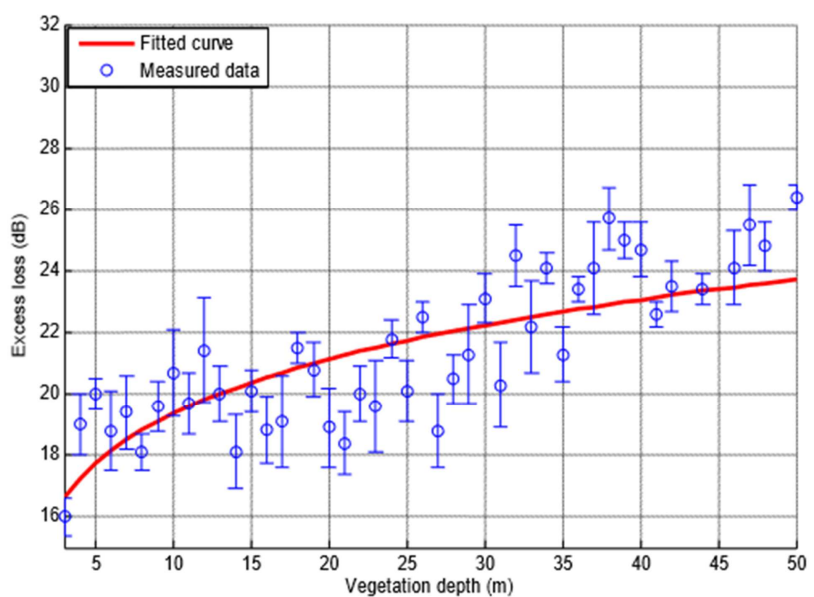

Figure 11. Excess loss for 'into geometry' fitted with a curve using the combined data at $3.5 \mathrm{GHz}$.

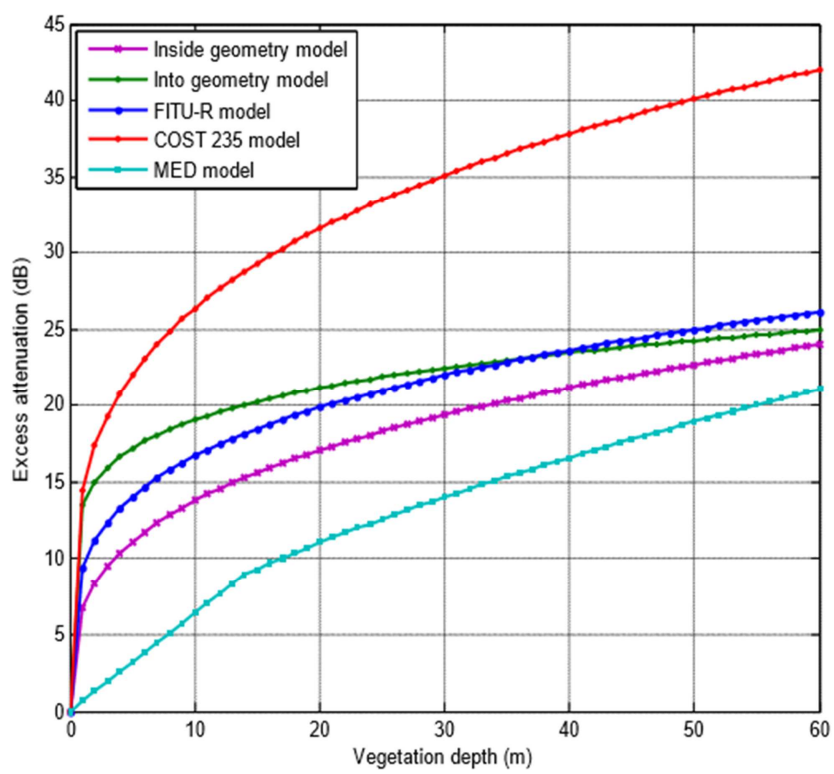

Figure 12. New prediction models for into and inside woodland compared with known empirical models at $3.5 \mathrm{GHz}$.

\section{Discussion}

In These measurements interaction of radio waves with vegetation structure going to excess attenuation. Dependence of the excess loss on foliage density appears in results shown in figures 6 and 8 . The full leaf recorded a loss of $8 \mathrm{~dB}-16 \mathrm{~dB}$ even at a shorter foliage depth while the latter for partial foliage recorded a loss of $2.4 \mathrm{~dB}-7 \mathrm{~dB}$ at a higher depth. Figure 7 shows different antenna height effects relative to the vegetation. Since the research work is still at a First stage, making a concluding remark would be premature for now. It is therefore hoped that upon completion, the contribution of each of these component parts (foliage density, path geometry, antenna height etc) to the excess loss would be well characterized. From this review, it appears that radio waves obstructed by vegetation suffer losses beyond free space. These losses depend on the frequency and depth of vegetation. Other factors are the type of tree, whether the trees are leafy or leafy, dry or wet, static or dynamic, etc. Accurate modeling of this excessive loss is highly desirable for wireless operators to serve as a useful tool in RF planning that to ensure a good quality of service (QoS) optimization of cellular coverage and the availability link point to point the communication. Several empirical, semi-empirical and analytical models have been developed to estimate the loss of propagation in vegetation. They have identified some disadvantages with these models: the empirical models are limited to specific measurements and give no indication of the physical processes involved in the propagation, while the semi-empirical models do not include the dynamic effects of the channel in its formulation. For example, the DG model is a parametric equation in which it is said that the loss of vegetation decreases with the increase of the frequency, canceling the wave propagation behavior in the vegetation, which increases the losses as the frequency increases; Analytical models have been demonstrated to provide more 
accurate predictions, but their formulation and validation is often dependent on experimental investigations, such as the RET model. This indicates that for the development of any model of reliable prediction of vegetation loss, experimental research is inevitable. Moreover, current analytical models have assumed the homogeneity of trees, which contrasts with the exact nature.

\section{Conclusion}

To verify the validity of the new models in equations 14 and 15, comparisons were made with well-known empirical models (FITU-R, MED and COST 235) and the results are presented in the plots

From the graphs in Figure 12, FITU-R gave the best combination of the newly formulated models. The values of the RMS into geometry are $1.6 \mathrm{~dB}$ at $3.5 \mathrm{GHz}$. The inside geometry measures RMS value of $2.6 \mathrm{~dB}$ at $3.5 \mathrm{GHz}$. (FITUR) showed good predictive ability with our measurement data. This is due to the fact that its formulation (FITU-R) is based on data measured from several sites with different geometries, tree types, and plants depth reduced by less than $120 \mathrm{~m}$ (Meng et al 2009). At reception heights of 2.5 to 4.0 $\mathrm{m}$, the model has always shown good adaptability to measurement data. On the other hand, COST 235 overestimated the expected loss at the height of the antenna used in the experiment. However, a good fit to the measurement data was observed (in COST 235) at an antenna height of about $5 \mathrm{~m}$ and above. It depends on the relative heights of the trees and the starting points of the branches. In most cases, the MED model underestimated the losses but shows a good fit when the height of the antenna is at the trunk level. All the curves in Figure 12 show a larger variable gradient in the MED model. In the first 10 meters of depth, the into geometry shows a higher attenuation value of about 5 $\mathrm{dB}$ to $7 \mathrm{~dB}$ above the expected loss of inside geometry. This loss difference gradually decreases to about $2 \mathrm{~dB}$ at $50 \mathrm{~m}$ depth and flattens at greater depths. The high attenuation values provided at the initial depth of the into geometry are a reflection of the LOS block in the incident plane. A few meters from the transmitting point of the inside geometry, the LOS is always present. With regard to into geometry, the intensity of the signal decreases more rapidly with the depth of vegetation than the field propagated using the inside geometry.

\section{References}

[1] Al-Nuaimi M. O and Hammoudeh A. M (1994): "measurements and predictions of attenuation and scatter of microwave signals by trees", IEE Proc. Microw. Antennas propag., Vol. 141, No 2, pp 70-76.

[2] Al-Nuaimi M. O and Stephens R. B. L (1998): "measurement and prediction model optimization for signal attenuation in vegetation media at centimeter wave frequencies", IEE Proc. Microw. Antennas propagate. Vol. 145. No. 3, pp. 201-206, June 1998.

[3] Ndzi D, Savage N and Stuart K (2005); "Wideband signal propagation through vegetation". XVII, GA of URSI, Delhi India.

[4] Meng Y. S, Lee Y. H and Chong B. N (2009): "The effects of tropical weather on radio wave propagation over foliage channel", IEEE Trans. Veh. Technol., Vol. 58, No 8, pp 4023 4030 .

[5] Meng Y. S, Yee Hui Lee and Boon Chong Ng (2009); "Empirical near proud path loss modeling in a forest at VHF and UHF bands", IEEE transactions on antennas and propagation. Vol. 57, No. 5, May 2009.

[6] Cheffena M and Ekman T (2009): "Dynamic model of Signal Fading due to swaying vegetation", Journal of wireless communications and networking. doi:10.1155/2009/306876.

[7] Tamir T, (1967); "On-radio-wave propagation in forest environments", IEEE trans. On Antenna and Propagation, AP15, (6), pp 806-817.

[8] Weissberger M. A, (1982); An initial critical summary of models for predicting the attenuation of radio waves by trees", Electromagnetic compatibility analysis center, Annapolis, Maryland. Final report.

[9] CCIR, "influences of terrain irregularities and vegetation on troposphere propagation", CCIR report, 235-236, Geneva, 1986.

[10] COST 235, "Radio propagation effects on next-generation fixed-service terrestrial telecommunication systems," final report, Luxembourg, 1996.

[11] Michael A. O, "Further investigation into VHF radio wave propagation loss over long forest channel", International Journal of Advanced Research in Electrical, Electronics and Instrumentation Engineering. Vol. 2, Issue 1, pp. 705-710, January 2013. 